\title{
Géolinguistique
}

16 | 2016

Varia

\section{Claudio Wagner, El Atlas Lingüístico y Etnográfico de Chile (ALECh) por regiones}

Universidad Austral, Valdivia

Pilar García Mouton

\section{OpenEdition}

\section{Journals}

Edición electrónica

URL: http://journals.openedition.org/geolinguistique/489

DOI: $10.4000 /$ geolinguistique.489

ISSN: 2650-8176

Editor

UGA Éditions/Université Grenoble Alpes

Edición impresa

Fecha de publicación: 1 diciembre 2016

Paginación: 255-260

ISBN: 978-2-84310-342-1

ISSN: 0761-9081

\section{Referencia electrónica}

Pilar García Mouton, «Claudio Wagner, El Atlas Lingüístico y Etnográfico de Chile (ALECh) por regiones »,

Géolinguistique [En línea], 16 | 2016, Publicado el 15 febrero 2019, consultado el 30 octubre 2020. URL http://journals.openedition.org/geolinguistique/489; DOI : https://doi.org/10.4000/geolinguistique. 489 


\title{
Claudio Wagner* El Atlas Lingüístico y Etnográfico de Chile (ALECh) por regiones
}

\author{
por \\ Pilar García Mouton \\ Instituto de Lengua, Literatura y Antropología (CSIC), Madrid
}

El Atlas Lingüístico y Etnográfico de Chile (ALECh) por regiones dirigido por Claudio Wagner y realizado con un equipo de investigadores - Victoria Espinosa, Patricia Uribe (para el ALECh Norte), Gustavo Rodríguez, Luis Tecas (para el ALECh Centro), Carlos Ramírez, María Catrileo, Raúl Caamaño (para el ALECh Sur), y Claudio Wagner, Eduardo Roldán, Claudia Rosas (para el ALECh Sur-Austral) - representa la conclusión de un atlas nacional en un país como Chile, donde varias importantes empresas geolingüísticas quedaron en la fase de proyecto y otras no llegaron a concluirse.

En 1996, al comenzar su colaboración sobre Chile en el Manual de dialectología hispánica. El Español de América, dirigido por Manuel Alvar, escribía Claudio Wagner: «No es fácil dar cuenta de la variación del español hablado en Chile cuando la información de que se dispone es escasa y por añadidura dispersa» y señalaba que hasta entonces «la historia de la zonificación propuesta para Chile adolece de mayores dificultades que la propuesta para Hispanoamérica», porque no se había hecho a partir de rasgos lingüísticos, de modo que el problema continuaba abierto, «ya que sólo investigaciones globales del tipo de los atlas lingüísticos pueden garantizar materiales precisos que permitan establecer científicamente esas deseadas zonas dialectales». Este Atlas Lingüístico y Etnográfico de Chile por regiones responde a estas necesidades científicas.

* Universidad Austral, Valdivia (Chile). 
Cuando Claudio Wagner escribió aquel capítulo sobre el español de Chile, acababa de terminar las encuestas previstas para Chile en el Atlas Lingüístico de Hispanoamérica, dirigido por Manuel Alvar y Antonio Quilis, con sus colegas Gustavo Rodríguez, Eduardo Roldán y Luis Tecas, miembros del equipo que había trabajado en el Atlas lingüístico y etnográfico del Sur de Chile de Guillermo Araya. Esas encuestas, hechas entre 1993 y 1995, sirvieron de estímulo para poner en marcha, con la ayuda del CONICYT, las tareas del Atlas Lingüístico y Etnográfico de Chile por regiones.

Las iniciativas de Geografía Lingüística planteadas en Chile tuvieron hasta ahora, como apunta Alfredo Matus en su Prólogo, «mala estrella [...] representada por sus tareas inconclusas, debidas a circunstancias lamentables». Una de las primeras iniciativas, el primer atlas regional americano, fue el Atlas Lingüístico y Etnográfico del Sur de Chile (1973), el ALESUCh, que dirigió Guillermo Araya, con Constantino Contreras, Claudio Wagner y Mario Bernales, cercano metodológicamente al Atlas Lingüístico y Etnográfico de Andalucía, de Manuel Alvar, Antonio Llorente Maldonado y Gregorio Salvador. De los cinco volúmenes previstos solo apareció el primero en 1968. La obra se interrumpió, y lo mismo ocurrió con el proyecto complementario, el Atlas Lingüístico-Etnográfico del Norte de Chile, dirigido por Ángel Araya, del que solo se editó una Muestra cartográfica del Léxico del cobre de 51 mapas en 1985 (ALeNoCh. Muestra cartográfica. Léxico del cobre. II. Región Chile, Antofagasta, Universidad del Norte). También existe un pequeño Atlas Lingüístico de Parinacota de Magdalena Contardo y Victoria Espinosa, cuya experiencia ha tenido que ser útil para las encuestas en la zona norte.

En 1988 Claudio Wagner publicó los criterios de elaboración de este Atlas Lingüístico y Etnográfico de Chile por regiones en la revista Estudios Filológicos. Señalaba que suponía el tercer intento de hacer un atlas lingüístico nacional, un atlas de gran dominio en la línea de los grandes atlas nacionales europeos, teniendo en cuenta que había habido un primer intento de Rodolfo Oroz a principios de los años 1940 y otro de Gastón Carrillo en 1967. Este tercer intento se pensó desde una experiencia que le ha asegurado bases sólidas. Aunque Wagner tuvo presentes los planteamientos que llevaron en su día a Albert Dauzat a diseñar el Nouvel Atlas linguistique de la France par régions (NALF), una suma de atlas regionales que permitiera, con su parte común, construir un atlas general, el ALECh no sigue la estructura de un atlas general hecho por regiones.

Sin separarse de la tradición geolingüística, el ALECh incorpora novedades metodológicas, es un atlas lingüístico nacional, dividido por regiones, urbano y rural, con una vertiente específicamente costeña. En el 
marco de la tradición, mantiene el interés por las cosas junto a las palabras, es también un atlas etnográfico, heredero de los atlas europeos.

El ALECh utiliza cuestionarios complementarios con una parte común dedicada al léxico general, 804 cuestiones que se preguntaron en todas las localidades. A esa parte común se añadieron 173 preguntas más en 28 ciudades para recoger el léxico urbano; 593 preguntas en 154 localidades para reunir el léxico rural, y 82 preguntas en 34 localidades costeñas para obtener el léxico marítimo.

De este modo, un solo atlas de gran dominio estudia los diferentes tipos de población que había que considerar en la geografía lingüística chilena. Y, por razones prácticas, muy relacionadas con aspectos históricos, geográficos, culturales y demográficos, el ALECh divide el territorio nacional en cuatro grandes zonas: la Norte, con 49 puntos de encuesta; la Centro, con 60; la Sur, con 59 y la Sur-Austral, con 48, si bien no establece cuestionarios diferentes para cada una de ellas. Serán los estudios que se hagan a partir de este atlas los que muestren si estas zonas reflejan realmente diferencias dialectales.

El equipo hizo las encuestas en tres años a partir de 1997, un tiempo récord. En 2012 estaba terminada la edición convencional, pensada para imprimirse en papel y todavía sin materializar, y entre 2014 y 2015 se preparó la actual edición electrónica.

Llegados a este punto cabría plantear una reflexión sobre lo conveniente de editar los atlas como se ha venido haciendo tradicionalmente y las bondades de editar atlas con ayuda de las nuevas tecnologías. Actualmente coordino la edición en soporte electrónico del Atlas Lingüístico de la Península Ibérica (ALPI) de Tomás Navarro Tomás en <www.alpi. csic.es>; publiqué en 2003 con Francisco Moreno Fernández el Atlas Lingüístico y Etnográfico de Castilla-La Mancha (ALeCMan), disponible en $<$ www.linguas.net/alecman>, lo mismo que el Atlas Dialectal de Madrid (ADiM), <adim.cchs.csic.es>, que codirijo con Isabel Molina Martos. Sin embargo, inquieta pensar en qué pasará cuando no estemos detrás de la página web actualizándola o si se quedara sin mantenimiento, si un atlas en soporte informático dejará de poder leerse cuando los programas evolucionen y no haya interesados en volcarlo de un soporte antiguo a un formato nuevo.

Ahora bien, las ventajas de un atlas informático en línea son evidentes, ya que lo hacen accesible desde cualquier computadora desde los lugares más alejados de las bibliotecas especializadas y, además, las posibilidades de una edición exhaustiva, sin límites de espacio, de toda la información recogida en las encuestas, son mucho mayores. Por otra parte, imprimir en papel exige un cartografiado a escala, y para un país como Chile, con unas 
características geográficas tan marcadas, la dificultad para resulta obvia para poder ver, leer un mapa lingüístico de una ojeada. El Atlas Lingüístico de Chile, al estar en soporte informático, permite ampliar y minimizar sus mapas para hacer visible la información proporcionada por los informantes elegidos para representar a los hablantes de 216 localidades chilenas, rurales, urbanas - en las 28 ciudades se contrastan dos niveles de habla, el habla culta y la popular - y de la costa.

El ALECh es un atlas amigable que no está pensado solo para especialistas, lo que se advierte desde el primer momento, porque combina respuestas en alfabeto fonético con respuestas en ortografía semiconvencional y con símbolos. Al pasar el ratón sobre un punto, aparece el nombre de la localidad; encima de un símbolo o de una transcripción fonética, aparece la forma ortográfica. En el margen izquierdo se abre un texto con información sobre el punto de encuesta en cuestión, foto incluida, y los informantes que proporcionaron las respuestas. Y, debajo del título del mapa, se encuentran la forma en que se preguntó y las correspondencias de la cuestión en algunos otros atlas hispánicos.

Cinco mapas preliminares sirven para contextualizar los contenidos del ALECh, y el grueso de los mapas lingüísticos se puede localizar a través de dos índices complementarios: un Índice temático, que sigue la estructura del cuestionario, y un Índice alfabético con el nombre de los mapas en mayúscula y todas las variantes registradas en minúscula, lo cual es novedoso y útil.

Además de los 216 puntos en suelo chileno, la red de encuesta añade nueve puntos de encuesta fuera del país para detectar posibles influencias externas: uno en Perú, Tacna; otro en Bolivia, Charaña; el resto, siete puntos argentinos: Mendoza, Codihué, Bariloche, Trevelin, Los Antiguos, Río Gallegos y Río Grande. En las ciudades, con respuestas de nivel alto y nivel bajo separadas por una barra vertical, el léxico urbano supone una novedad respecto a los cuestionarios tradicionales (Pueblo, Comercio, Comunicaciones, Ocupaciones, Política, Administración pública, Economía social, Educación).

Cuando los datos de encuesta no son suficientemente interesantes o completos para merecer un mapa propio, se incorporan como micromapas que registran la uniformidad de ciertas denominaciones, como el micromapa de acelga y brócoli, el de achicoria, el de alcachofa (solo dos casos de alcaucil), el de damasco, el de espárrago, el de sandía y melón, el de palta 'aguacate', y el de celular 'teléfono portátil'. Las láminas de texto reúnen información complementaria, dibujos y contenidos aprovechables cercanos a las notas. 
El ALECh ofrece información interesante desde el punto de vista fonético y morfológico, pero es en el léxico donde se percibe la convivencia del español con las lenguas indígenas. El mapa 676, por ejemplo, que reúne los nombres del renacuajo, renacuajo, guarisapo en el norte, deja ver cómo más al sur este tipo de denominaciones son refugio de nombres indígenas: en el centro, pirihuín, pirihuini; en el sur coltrao, coltrahue, coltraíto y coihuilla, que se dan también en la zona sud-austral donde, sobre todo en Isla Grande y la costa adyacente, la respuesta general es colhuín. El mapa 118, El Demonio es de una riqueza llamativa: Satanás, el Satánico, Satán, don Sata, sobre todo en el norte, pero también Lucifer, Belcebú, Luzbel, Ángel Malo, el Malo, el Maligno, el Malulo, Maldito, el Cachu(d) o, Cacholargo, Cachivo, Colu(d)o, Cornudo, Cuernúo, el Cuco, el Amigo, el Patah largah, el Pata de cabro, el Patah de hilo, el Pata rajá, el Pata negra, el Boca de juego, el Colilargo, Cola de lima, el Caballero de los cachos, el Manos lanudas, etc. Interesa especialmente la distribución geográfica de el Diaulo, con algún caso aislado en el centro, casi siempre en el sur.

El género gramatical protagoniza algunos de estos mapas. No parece que en el mapa 975, Agua: género, el artículo en el agua suponga género masculino, lo mismo que ocurre con el azúcar en el mapa 977. Interesan los que marcan la dirección de los nuevos femeninos, como el mapa 991, Jefe (femenino) donde casi es general la jefa; el mapa 1008, Alcalde (femenino), que apunta la alcaldesa como uso casi general, con algún resto de la alcalde, o el micromapa de Director (femenino) que da directora. La fuerza de la analogía impresiona en el mapa 985, Nuera por la abundancia de yerna en la zona central, la más urbana del país.

Evidentemente el ALECh ofrece mucho más: mapas de fonética vocálica (901 Teatro), de fonética consonántica (136 Fútbol; 922 Dormido, -da); de fonética en préstamos, de morfología, de sintaxis... En este último aspecto se echa de menos la edición de las grabaciones libres, conversaciones y narraciones, que se hicieron al acabar cada encuesta, material fundamental para estudiar sintaxis, fonosintaxis y entonación en contexto.

El ALECh es fruto de una Geografía Lingüística tradicional actualizada. Es el momento de abordar la segunda etapa, que supone el estudio y la interpretación de estos mapas y su aprovechamiento desde todo tipo de enfoques: fonéticos, fonológicos, gramaticales, lexicológicos, lexicográficos, sintácticos, sociolingüísticos, etnográficos, históricos, de lenguas en contacto actual o pasado, etc. El subtítulo por regiones puede confundir a los especialistas, porque lo que se fragmenta en regiones es la presentación de un atlas nacional, no estamos ante atlas regionales diferentes. Esos atlas 
podrán hacerse a partir del ALECh, marco de cualquier estudio lingüístico posterior. El Atlas Lingüístico y Etnográfico de Chile por regiones le da por fin su lugar a Chile en la tradición geolingüística: en la tradición americana, en la latinoamericana, en la hispanoamericana y especialmente en la hispánica. 